\title{
ANÁLISE POR INJEÇÃO EM FLUXO COM DETECÇÃO AMPEROMÉTRICA DE MÚLTIPLOS PULSOS: POTENCIALIDADES E APLICAÇÕES
}

\author{
Wallans Torres Pio dos Santos \\ Departamento de Farmácia, Universidade Federal dos Vales do Jequitinhonha e Mucuri, 39100-000 Diamantina - MG, Brasil \\ Denise Tofanello Gimenes e Eduardo Mathias Richter* \\ Instituto de Química, Universidade Federal de Uberlândia, CP 593, 38400-089 Uberlândia - MG, Brasil \\ Lúcio Angnes \\ Instituto de Química, Universidade de São Paulo, CP 26077, 13560-970 São Paulo - SP, Brasil
}

Recebido em 29/11/10; aceito em 15/3/11; publicado na web em 5/5/11

\begin{abstract}
FLOW INJECTION ANALYSIS WITH MULTIPLE PULSE AMPEROMETRIC DETECTION: POTENTIALITIES AND APPLICATIONS. The potentialities and applications of the Multiple Pulse Amperometric detection (MPA) coupled with Flow Injection Analysis (FIA) are evaluated. Important aspects as cleaning and activation of electrode surface, indirect and simultaneous analysis of electroactive compounds and the use of the internal standard method for quantifications utilizing FIA-MPA are presented. The main parameters concerning the detection of electroactive analytes by multiple pulse amperometric detection in flowing solutions were also discussed. In addition, aspects such as flow rate, sample volume, application time of the potential pulses and instrumentation necessary for implementing of the method were also addressed.
\end{abstract}

Keywords: electrochemical detection; multiple pulse amperometry; flow injection analysis.

\section{INTRODUÇÃO}

O desenvolvimento de métodos analíticos que combinam menor tempo de análise, maior sensibilidade e seletividade, simplicidade de manuseio, baixo custo e potencialidade adequada para automação constitui-se num campo de pesquisa amplo e em constante expansão na área de Química Analítica. Nesse contexto, a análise por injeção em fluxo, FIA - Flow Injection Analysis, tem sido explorada com sucesso na análise de grande diversidade de substâncias, proporcionando várias vantagens, tais como, uso de instrumentação versátil, baixo custo dos componentes do sistema, elevada frequência de amostragem, consumo reduzido de reagentes e amostras e, consequentemente, redução na geração de resíduos. ${ }^{1-7}$ Além disto, o sistema FIA pode ser automatizado, minimizando os riscos de intoxicação do analista devido a uma menor exposição aos reagentes e amostras em comparação aos procedimentos manuais. Os primeiros trabalhos empregando FIA foram propostos por Rüzicka e Hansen, em 1975, como um novo conceito de análises químicas. ${ }^{8}{ }^{89}$ No primeiro artigo, os autores descrevem o sistema FIA acoplado a detectores de absorção molecular. ${ }^{8}$ Três anos depois, os mesmos autores fazem uma revisão sobre os dez trabalhos que haviam desenvolvido anteriormente, abordando a teoria e as tendências da técnica. ${ }^{9}$ Posteriormente, uma diversidade de técnicas foram exploradas, cabendo destacar a absorção atômica, ${ }^{10,11}$ fluorescência, ${ }^{12,13}$ quimiluminescência, ${ }^{14,15}$ potenciometria, ${ }^{16,17}$ voltametria, ${ }^{18,19}$ amperometria, ${ }^{20,21}$ entre outros.

A detecção eletroquímica pode oferecer vantagens frente a outros sistemas de detecção, como baixo custo de instrumentação, simplicidade de aplicação e minimização de etapas de preparação de amostras..$^{3,22-25}$ Adicionalmente, diversas espécies a serem analisadas podem ser pré-concentradas sobre eletrodos, tanto por processos de

*e-mail: emrichter@iqufu.ufu.br adsorção como por eletrodeposição. Os detectores eletroquímicos mais utilizados em sistemas em fluxo são os amperométricos, que foram adotados em grande parte dos trabalhos descritos na literatura. ${ }^{3}$ Nesta técnica, o eletrodo de trabalho (ou sensor eletroquímico) é mantido num potencial constante adequado, no qual ocorre a oxidação ou redução eletroquímica de compostos eletroativos de interesse. As principais vantagens da detecção amperométrica convencional acoplada a sistemas FIA em comparação com os métodos voltamétricos convencionais são maior sensibilidade (devido ao enorme incremento no transporte de massa proporcionado pelo fluxo da solução), menores efeitos de contaminação da superfície do eletrodo de trabalho, uma vez que o tempo de contato da amostra e dos produtos do processo redox com o eletrodo é muito menor do que em processos estacionários e, principalmente, a presença de corrente capacitiva negligenciável (muito pequena) instantes depois do início do experimento (aplicação de um único potencial em função do tempo), o que favorece medições de correntes faradaicas baixíssimas (e, consequentemente, de baixas concentrações). ${ }^{26}$ No entanto, é importante destacar que a medição de correntes muito baixas $\left(<10^{-9} \mathrm{~A}\right)$ pode exigir cuidados adicionais, como o uso de uma gaiola de Faraday, por exemplo.

Uma limitação associada a esse modo de detecção é a estabilidade do sinal eletroquímico em função do tempo quando determinados compostos são analisados, comprometendo a repetibilidade da resposta e a reprodutibilidade dos resultados. A estabilidade do sinal eletroquímico é governada pela taxa de transferência de carga (elétrons) entre o eletrodo e a espécie eletroativa (presente na interface eletrodosolução), que por sua vez depende das condições da superfície do eletrodo. A superfície do eletrodo pode ser modificada gradativamente durante o experimento, dependendo do tipo de composto que está envolvido no processo eletroquímico. Quando uma espécie eletroativa e/ou produto de reação adsorve ou eletropolimeriza na superfície do eletrodo de modo irreversível ou quase irreversível, pode ocorrer uma 
contaminação e/ou passivação do eletrodo. Este problema pode ser verificado, por exemplo, na determinação eletroquímica de fenóis e seus derivados. ${ }^{27}$ A passivação e/ou contaminação da superfície do eletrodo pode afetar a taxa de transferência de carga entre o eletrodo e $\mathrm{o}$ analito, assim como produzir sinais eletroquímicos devido aos produtos adsorvidos, que interferem no sinal eletroquímico de interesse.

Outra limitação da detecção amperométrica convencional (usando um único eletrodo de trabalho) é a impossibilidade da determinação simultânea de compostos eletroativos com potenciais redox distintos em determinadas condições experimentais. Quando a diferença de potencial envolvida em ambos os processos eletroquímicos é suficientemente grande, superior a $0,1 \mathrm{~V},{ }^{28}$ é possível contornar esta limitação realizando-se medidas do sinal amperométrico em potenciais distintos $\left(\mathrm{E}_{1}\right.$ e $\left.\mathrm{E}_{2}\right)$ sequencialmente. Na injeção da primeira alíquota da amostra e aplicando o potencial $\mathrm{E}_{1}$, somente uma das espécies é reduzida ou oxidada, sendo o sinal amperométrico proporcional à concentração desta espécie. Em seguida, com a injeção de uma segunda alíquota da mesma amostra e aplicação do potencial $\mathrm{E}_{2}$, ambas as espécies sofrem processo de oxidação ou redução. $\mathrm{O}$ sinal amperométrico diferencial corresponderá à concentração da segunda espécie. Além disto, essa análise pode ser realizada simultaneamente utilizando-se uma célula eletroquímica em fluxo com dois eletrodos de trabalho em paralelo, um fixado no potencial $\mathrm{E}_{1}$ e outro em $\mathrm{E}_{2} \cdot{ }^{29} \mathrm{~A}$ utilização de uma rede de microeletrodos associada a tratamentos quimiometricos também é descrita na literatura para determinação simultânea de dois ou mais analitos. ${ }^{30}$ No entanto, cabe ressaltar que nestes casos, um bipotenciostato ou multipotenciostato são exigidos para controlar o potencial de cada eletrodo de trabalho, o que aumenta a complexidade (e consequentemente o custo) da instrumentação requerida para realização da medida. Além disto, os softwares (atualmente disponíveis) não permitem que se utilize mais de um eletrodo simultaneamente na amperometria pulsada, o que compromete a limpeza eletroquímica, visto que a mesma não pode ser programada no transcorrer do experimento.

Como já mencionado, a detecção amperométrica acoplada a sistemas em fluxo pode ser implementada no modo pulsado, na qual pulsos de potenciais são aplicados ao eletrodo de trabalho sequencialmente e continuamente, em função do tempo. Esse modo de detecção é conhecido na literatura como detecção amperométrica pulsada, PAD - Pulsed Amperometric Detection. A PAD geralmente contorna de forma simples as limitações da detecção amperométrica convencional em relação à estabilidade do sinal eletroquímico em função do tempo. Esta técnica permite que a resposta do eletrodo apresente estabilidade por um período de tempo maior devido à periódica limpeza eletroquímica realizada durante o experimento, evitando ou diminuindo a contaminação da superfície do eletrodo.,31-33

A técnica PAD é disponibilizada em diversos softwares comerciais de potenciostatos do mercado. O número de pulsos de potenciais aplicáveis varia entre os diferentes equipamentos comercializados (mínimo de 2 até 6 pulsos de potenciais). Porém, a aquisição do sinal de corrente é normalmente restrita a um único pulso de potencial. Uma alternativa bastante vantajosa para esta técnica somente é disponibilizada (segundo nosso conhecimento) pelo software que controla os potenciostatos comercializados pela empresa Metrohm - Eco Chemie (software GPES). Este software permite a aplicação de até 10 pulsos de potenciais com a possibilidade de aquisição da corrente em função do tempo em cada pulso de potencial (o que corresponde à aquisição de 10 amperogramas distintos "simultaneamente"). A técnica é conhecida como amperometria de múltiplos pulsos, MPA - Multiple Pulse Amperometry. O tempo mínimo de aplicação de cada pulso é de 30 ms (limitação do software).

Na literatura são descritos diversos trabalhos que aplicam a detecção MPA ou PAD associados a sistemas em fluxo, com a finalidade de melhorar o desempenho de métodos analíticos empregados em amostras de origens diversas. Grande parte dessas aplicações tem o intuito de realizar a limpeza e/ou ativação da superfície do eletrodo de trabalho. Ainda que pouco explorada, alguns artigos descrevem a utilização da MPA em FIA para detecção indireta ou simultânea de analitos eletroativos. Diante deste contexto, o presente trabalho apresenta uma revisão sobre os artigos disponibilizados na literatura que utilizam a amperometria pulsada para melhorar o desempenho dos métodos analíticos empregados em diversas áreas. Aspectos relevantes e pouco explorados na literatura sobre o uso da técnica de amperometria de múltiplos pulsos como detector em sistemas em fluxo também serão discutidos.

\section{LIMPEZA E ATIVAÇÃO DA SUPERFÍCIE DO ELETRODO ATRAVÉS DA MPA}

As técnicas amperométricas baseadas na MPA podem contornar de uma forma simples os problemas relacionados à contaminação e/ou passivação da superfície do eletrodo de trabalho. O uso de dois pulsos de potenciais (aplicação mais comum) possibilita somente a detecção da espécie de interesse (em $\left.\mathrm{E}_{1}\right)$ e a periódica limpeza eletroquímica do eletrodo ( $\mathrm{em}_{2}$ ) durante o transcorrer do experimento. No entanto, em muitos casos é necessária a aplicação de três ou mais pulsos de potenciais para realizar, além da limpeza eletroquímica, a ativação constante do eletrodo de trabalho, com intuito de alcançar uma boa estabilidade do sinal eletroquímico ao longo da medida. ${ }^{34}$ Isto pode ser explicado pelo fato da ativação produzir, em alguns processos, produtos intermediários, ou causar modificações na superfície do eletrodo, que favorecem os processos de transferência de carga entre o eletrodo e as espécies eletroativas de interesse, resultando numa maior sensibilidade na resposta do detector. Se a superfície do eletrodo for mantida limpa e eletroquimicamente ativa durante o experimento, aumenta consideravelmente a possibilidade de obtenção de respostas estáveis, reprodutíveis e relacionáveis com a concentração do analito na solução (faixa linear de trabalho).

A detecção amperométrica pulsada (PAD ou MPA) foi utilizada por diversos autores acoplada a sistemas FIA, ${ }^{3,31,35-41}$ eletroforese capilar (EC) $)^{42-44}$ e cromatografia líquida de alta eficiência (CLAE), correspondendo esta última ao maior número de artigos disponibilizados na literatura sobre o assunto..$^{33,34,45-57}$ Nestes sistemas, a principal função da detecção pulsada foi promover a limpeza eletroquímica constante e ativação da superfície do eletrodo de trabalho. A amplitude, polaridade e tempos de aplicação dos pulsos de potenciais, de detecção, limpeza e ativação podem ser variados, no sentido de se garantir a maior sensibilidade e estabilidade do sinal eletroquímico para uma diversidade de analitos. Embora os métodos de limpeza eletroquímica sejam muito úteis para se garantir a estabilidade da resposta dos detectores amperométricos durante o tempo de medida, estes procedimentos não eliminam a limpeza mecânica do eletrodo após certo período, especialmente quando as espécies analisadas adsorvem fortemente sobre a superfície desse eletrodo. Em alguns casos, a adsorção é irreversível eletroquimicamente e o uso de pulsos de potenciais de limpeza pode apresentar limitações.

Os primeiros trabalhos sobre amperometria pulsada foram apresentados no final da década de 70 e início de $80 .{ }^{58-61}$ Posteriormente, a partir de 1989, revisões sobre ampeometria pulsada foram disponibilizadas na literatura com enfoque nos seguintes temas: reações anódicas diretas e indiretas, ${ }^{62}$ detecção de carboidratos, aminas e compostos sulfurosos por cromatografia de íons, ${ }^{63}$ detecção de carboidratos e glicopeptídeos por eletroforese capilar, ${ }^{64}$ determinação de oligossacarídeos em alimentos e produtos agrícolas, ${ }^{65}$ análise de misturas de amino-carboidratos por cromatografia de troca iônica ${ }^{66}$ e determinação de derivados fenólicos usando eletrodo rotatório. ${ }^{67}$ 
Adicionalmente, foram publicados artigos de revisão que tratam da detecção eletroquímica de uma forma geral, contendo um tópico dedicado à amperometria pulsada. ${ }^{23,42,43,61,68-83}$ Estes trabalhos apresentam uma ampla revisão sobre aplicações da PAD ou MPA em procedimentos em batelada, eletroforese capilar e cromatografia líquida. No entanto, poucos trabalhos relatam o uso da MPA na análise simultânea ou indireta de analitos eletroativos empregando FIA. Além disto, aspectos relevantes em relação à variação da vazão, volume de injeção e tempo de aplicação do pulso de potencial raramente são abordados. Portanto, o presente trabalho reúne temas pouco abordados em trabalhos de revisão anteriores.

\section{ANÁLISES DE COMPOSTOS ELETROATIVOS ATRAVÉS DA MPA EM FIA}

Apesar da técnica de MPA ou PAD ser conhecida no meio científico como sistema de detecção em FIA, esta técnica, até o momento, foi pouco explorada como detector no modo gerador-coletor para espécies que apresentam comportamento redox. Considera-se como modo gerador-coletor quando uma espécie é oxidada ou reduzida em um pulso de potencial $\left(\mathrm{E}_{1}\right)$ e o produto gerado nesta etapa é detectado em um pulso de potencial subsequente $\left(\mathrm{E}_{2}\right)$. Neste caso, o analito pode ser detectado indiretamente (detecção do produto gerado em um pulso de potencial anterior) com a possibilidade de supressão do sinal gerado por possíveis interferentes presentes na amostra, os quais são eletroativos somente em $E_{1}$. Outro procedimento pouco explorado na MPA em FIA é a análise simultânea de compostos eletroativos que possuam potenciais de oxidação ou redução distintos em determinadas condições experimentais, minimizando a desvantagem apresentada pela amperometria convencional em FIA frente aos métodos cromatográficos. A seguir, serão discutidos os principais trabalhos apresentados na literatura que exploraram a MPA com finalidades analíticas.

\section{Análise indireta}

A análise indireta de compostos eletroativos utilizando a detecção MPA em FIA pode ser considerada quando se quantifica uma espécie através do monitoramento do produto de oxidação ou redução gerado na superfície de um eletrodo de trabalho. Alguns trabalhos foram localizados na literatura onde esta técnica é usada. Um desses artigos foi apresentado por Kubota et al.,$^{84}$ onde os autores descrevem a determinação de cisteína sob eletrodo de trabalho de ouro com solução transportadora de tampão fosfato $0,10 \mathrm{~mol} \mathrm{~L}^{-1}$ $(\mathrm{pH}=10,0)$. A sequência de aplicação dos pulsos de potenciais para detecção de cisteína através da técnica MPA em FIA foi a seguinte: +0,10 V/700 ms: oxidação da cisteína e adsorção do produto gerado na superfície do eletrodo de trabalho (ouro sem modificação química); - $-0,60$ V/30 ms: detecção e quantificação indireta de cisteína pela redução do produto adsorvido; $-1,30 \mathrm{~V} / 2000 \mathrm{~ms}$ : renovação e limpeza da superfície do eletrodo de trabalho. $\mathrm{O}$ limite de detecção calculado para cisteína foi $5,0 \times 10^{-7} \mathrm{~mol} \mathrm{~L}^{-1}$.

Recentemente, outros trabalhos foram publicados onde a determinação indireta foi explorada usando esta técnica. ${ }^{85,86}$ Gimenes et al. descrevem a determinação indireta de dopamina em FIA na presença de altas concentrações de ácido ascórbico, aplicando a seguinte sequência de pulsos de potenciais sob um eletrodo de trabalho de carbono vítreo: $+0,80 \mathrm{~V} / 700 \mathrm{~ms}$ : a dopamina e o ácido ascórbico são oxidados, gerando, respectivamente, $o$-dopaminoquinona (composto eletroquimicamente reduzível) e ácido de-hidroascórbico (composto normalmente inativo eletroquimicamente); +0,35 V/30 ms: quantificação indireta da dopamina pela redução eletroquímica da $o$-dopaminoquinona; $0,00 \mathrm{~V} / 500 \mathrm{~ms}$ : pulso de potencial aplicado para promover a limpeza eletroquímica da superfície do eletrodo de carbono vítreo. Neste estudo, o limite de detecção calculado para dopamina foi $50 \mathrm{nmol} \mathrm{L}^{-1}$ na presença de uma concentração de ácido ascórbico 5000 vezes maior $\left(1,0 \mathrm{mmol} \mathrm{L}^{1}\right)$.

No outro trabalho, dos Santos et al. apresentam a possibilidade da análise tanto direta quanto indireta do pesticida paration usando MPA acoplada à FIA. O pesticida foi quantificado em três potenciais distintos utilizando-se eletrodo de carbono vítreo em meio de ácido cítrico $0,05 \mathrm{~mol} \mathrm{~L}^{1}$. A função e a sequência dos pulsos de potenciais aplicados foi a seguinte: $-0,50 \mathrm{~V} / 30 \mathrm{~ms}$ : o grupo nitro presente na estrutura do paration é reduzido $\left(-\mathrm{NO}_{2}\right)$ ao grupo hidroxilamina (-NHOH). Neste pulso de potencial, o paration pode ser diretamente quantificado; +0,40 V/30 ms: o grupo hidroxilamina é oxidado ao grupo nitroso (-NO), podendo o pesticida ser quantificado indiretamente neste pulso de potencial; $-0,10 \mathrm{~V} / 30 \mathrm{~ms}$ : o grupo nitroso é reduzido ao grupo hidroxilamina, possibilitando uma segunda análise indireta neste pulso de potencial. O limite de detecção estimado é inferior a $1,0 \times 10^{-8} \mathrm{~mol} \mathrm{~L}^{-1}$ para determinação de organofosforados (paration) e derivados fenólicos.

Com o mesmo objetivo do trabalho anterior, porém utilizando uma célula eletroquímica sob agitação mecânica em substituição ao sistema FIA, Tormin et al. demonstraram a determinação direta e indireta da terc-butil-hidroquinona (TBHQ) em biodiesel por meio da técnica de MPA. ${ }^{87}$ Os seguintes pulsos de potenciais foram aplicados sobre eletrodo de trabalho de carbono vítreo versus $\mathrm{Ag} / \mathrm{AgCl}$ em meio de $\mathrm{HClO}_{4}$ 0,05 mol L//etanol (25/75): +0,70 V/300 ms: pulso de potencial aplicado para oxidação e quantificação direta da TBHQ; 0,00 V/100 ms: pulso de potencial aplicado para redução do produto formado pela oxidação da TBHQ em $0,70 \mathrm{~V}$, possibilitando a quantificação indireta do analito; - $-0,05 \mathrm{~V} / 1000 \mathrm{~ms}$ : pulso de potencial aplicado para constante limpeza eletroquímica da superfície do eletrodo de trabalho. O método proposto permite a determinação direta de TBHQ em biodiesel (após simples diluição no eletrólito suporte) com o limite de detecção estimado em $5 \mu$ mol L ${ }^{-1}$.

A literatura apresenta um número considerável de métodos eletroquímicos nos quais diversos compostos são quantificados. Porém, na maioria dos casos, eletrodos sólidos quimicamente modificados são utilizados para evitar a contaminação da superfície do eletrodo e/ou para obtenção de seletividade frente a outros compostos presentes nas amostras. O uso de um sistema FIA simples ou célula sob convecção com detecção amperométrica pulsada permitiu o uso de eletrodos sólidos sem modificação química com incremento na seletividade do método, sem perda na sensibilidade e reprodutibilidade dos resultados obtidos.

\section{Análise simultânea}

Em geral, a estratégia mais utilizada na determinação simultânea de compostos é o uso de uma coluna de separação posicionada num estágio anterior ao detector. No entanto, esse tipo de alternativa requer, na maioria dos casos, o uso de sistemas cromatográficos. Estes equipamentos, além de dispendiosos, ainda necessitam de uma etapa de preparação da amostra (geralmente bastante trabalhosa) para garantir seletividade ou sensibilidade (ou ambas) na detecção, o que pode comprometer o tempo da medida e produzir quantidades significativas de resíduos. A determinação simultânea em fluxo sem utilização de colunas de separação tem sido proposta em sistemas FIA com detecção espectrofotométrica ${ }^{88-96}$ Mesmo apresentando vantagens em alguns casos em relação aos métodos cromatográficos (custo inferior e maior frequência analítica), esta técnica também necessita de etapas de tratamento prévio da amostra para remoção de interferentes e, além disto, os dados gerados geralmente necessitam de um tratamento quimiométrico para que a quantificação simultâ- 
nea e seletiva seja possível. O uso da MPA acoplada à FIA para este fim pode apresentar vantagens em relação a tempo, simplicidade de aplicação, baixo custo e geração de resíduos em menor volume frente aos métodos descritos anteriormente.

O primeiro trabalho apresentado na literatura utilizando a MPA em FIA para análise simultânea de compostos eletroativos foi proposto por Surareungchai e coautores. ${ }^{97}$ Eles descrevem a análise simultânea de glicose e frutose com aplicação de quatro pulsos de potenciais usando um eletrodo de ouro modificado com Nafion em meio de $\mathrm{NaOH} 0,10 \mathrm{~mol} \mathrm{~L}^{1}$. Os pulsos de potenciais foram otimizados na seguinte sequência: $-0,50 \mathrm{~V} / 240 \mathrm{~ms}$ : o grupo aldeído presente na glicose é oxidado e usado para quantificação seletiva deste analito sem a interferência da frutose; +0,20 V/180 ms: o grupo aldeído presente na glicose é oxidado juntamente com os grupos alcoóis presentes tanto na glicose quanto na frutose. Dessa forma, como a frutose não possui a função aldeído em sua estrutura, a diferença entre as respostas amperométricas do primeiro pulso de potencial aplicado $(-0,5 \mathrm{~V})$ e o segundo pulso de potencial $(+0,2 \mathrm{~V})$ fornece proporcionalmente a resposta para quantificação da frutose sem a interferência da glicose; $+1,00 \mathrm{~V} / 180 \mathrm{~ms}$ : pulso de potencial usado para a limpeza da superfície do eletrodo de trabalho; - 0,80 V/300 ms: pulso de potencial usado para reativação da superfície do eletrodo de trabalho. O método foi usado na determinação de glicose e frutose em 5 amostras de frutas, com a obtenção de resultados similares por cromatografia líquida. Os limites de detecção foram calculados em 1,2 e 0,13 $\mathrm{mmol} \mathrm{L}^{-1}$ para glicose e frutose, respectivamente. Segundo os autores, é o primeiro trabalho no qual a técnica FIA com detecção amperométrica pulsada é usada para análise simultânea destes açúcares.

Recentemente, dos Santos et al. exploraram a MPA acoplada à FIA para análise simultânea de paracetamol e ácido ascórbico ${ }^{98}$ e, posteriormente, dipirona e paracetamol, ${ }^{99}$ ambos em formulações farmacêuticas. No primeiro trabalho, a seguinte sequência de pulsos de potenciais foi continuamente aplicada (de forma cíclica) em função do tempo: $+0,40 \mathrm{~V} / 100 \mathrm{~ms}$ : o ácido ascórbico é oxidado e quantificado sem a interferência de paracetamol; +0,65 V/100 ms: oxidação de ambas as espécies, no qual somente o produto eletroquimicamente gerado pelo paracetamol $(N$-acetil- $p$-benzoquinonaimina) é eletroativo na região catódica; $0,00 \mathrm{~V} / 100 \mathrm{~ms}$ : o produto gerado eletroquimicamente pela oxidação do paracetamol é reduzido e quantificado sem a interferência de ácido ascórbico; 0,05 V/600 ms: pulso de potencial usado para completa regeneração (limpeza) da superfície do eletrodo de trabalho. Neste estudo, o enfoque principal foi dado à frequência analítica do método (60 injeções/h), uma vez que na análise de formulações farmacêuticas, os limites de detecção não necessitam ser muito baixos. O método foi aplicado na análise de 6 amostras de formulações farmacêuticas, com a obtenção de resultados similares aos obtidos por metodologias desenvolvidas anteriormente. Além disto, os autores demonstram a possibilidade da análise indireta de paracetamol na presença de altas concentrações de ácido ascórbico (176 mg L $\left.\mathrm{m}^{-1}\right)$, com o limite de detecção calculado em $0,2 \mathrm{mg} \mathrm{L}^{-1}$, o que permite a determinação de paracetamol na presença de ácido ascórbico 880 vezes superior. Porém, o meio eletrolítico necessitou ser alterado para uma solução de ácido sulfúrico $0,10 \mathrm{~mol} \mathrm{~L}^{-1}$, pois nesse meio, a interferência provocada pela presença de altas concentrações do ácido ascórbico é eliminada. Essa interferência ocorre devido a uma reação química entre o ácido ascórbico e o produto de oxidação do paracetamol ( $N$-acetil- $p$-benzoquinonaimina), levando a um desvio negativo na curva analítica para análise indireta do paracetamol. Em meio ácido, essa reação é inibida e o desvio deixa de ser significativo.

No segundo artigo, os autores também usaram quatro pulsos de potenciais para determinar dipirona e paracetamol simultaneamente em meio de tampão acetato sobre eletrodo de trabalho de carbono vítreo, são eles: $+0,40 \mathrm{~V} / 100$ ms: oxidação e quantificação direta de dipirona sem a interferência de paracetamol; +0,65 V/100 ms: oxidação de ambos os analitos e, consequentemente, geração da $N$-acetil-p-benzoquinonaimina pela oxidação do paracetamol; $0,00 \mathrm{~V} / 200$ ms: quantificação indireta do paracetamol pela redução do produto gerado no pulso de potencial anterior; - 0,05 V/400 ms: limpeza eletroquímica da superfície do eletrodo de trabalho. Coincidentemente, os potenciais aplicados nos dois estudos são os mesmos, porém a aplicação do terceiro pulso de potencial para quantificação indireta de paracetamol exigiu um criterioso estudo do tempo de aplicação deste pulso de potencial. O problema para determinação indireta do paracetamol neste potencial foi provocado pelo fato do produto de oxidação da dipirona (gerado em $+0,65 \mathrm{~V}$ ) também gerar um produto eletroquimicamente ativo em potenciais mais negativos, implicando em uma interferência na análise indireta de paracetamol. O artifício utilizado para a detecção seletiva de paracetamol neste pulso de potencial foi a aquisição do sinal de corrente após $200 \mathrm{~ms}$ sob uma vazão de $1,5 \mathrm{~mL} \mathrm{~min}^{-1}$. Isto foi possível porque a corrente de redução gerada a partir dos produtos de oxidação do paracetamol e dipirona diminui à medida que o tempo de aplicação do pulso de potencial for aumentado. Como a corrente catódica para o produto de oxidação da dipirona possui menor intensidade, a corrente para este analito neste tempo (200 ms) cai a zero na interface eletrodo-solução e o sinal de corrente para a redução da $N$-acetil- $p$-benzoquinonaimina pôde então ser adquirido sem interferência da dipirona. A frequência analítica foi calculada em 45 injeções/h. O método foi testado com a análise de 5 amostras de formulações farmacêuticas e de uma mistura artificial, com a obtenção de resultados equivalentes aos obtidos por titulação iodométrica (dipirona) ou com solução de $\mathrm{Ce}^{4+}$ (paracetamol).

Outro trabalho utilizando a MPA em FIA é descrito por Medeiros et al.. Este trabalho apresenta a determinação simultânea de antioxidantes fenólicos (butil-hidróxi-anisol (BHA) e butil-hidroxitolueno (BHT)) de forma simples e rápida usando eletrodo de trabalho de diamante dopado com boro. ${ }^{100}$ Foram aplicados apenas dois pulsos de potenciais na seguinte sequência: $+0,85 \mathrm{~V} / 200 \mathrm{~ms}$ : oxidação e quantificação direta da BHA sem a interferência de BHT; +1,15 V/200 ms: oxidação de ambos os analitos (BHA e BHT). A quantificação da BHT foi obtida pela diferença entre as respostas amperométricas no segundo $(1,15 \mathrm{~V})$ e no primeiro pulso de potencial $(+0,85 \mathrm{~V})$. Cabe destacar que, nesse trabalho, não foram usados pulsos de potenciais para limpeza ou ativação do eletrodo de trabalho. A ausência desses potenciais é justificada pela alta estabilidade do eletrodo de diamante dopado com boro juntamente com o pré-tratamento catódico anteriormente realizado. O método foi aplicado na determinação de antioxidantes em amostras de maionese com obtenção de resultados semelhantes aos obtidos por cromatografia líquida. Os limites de detecção foram calculados em 0,030 e $0,40 \mu \mathrm{mol} \mathrm{L}^{-1}$ para BHA e BHT, respectivamente. A frequência analítica obtida foi de 63 injeções $/ \mathrm{h}$.

A técnica MPA também foi explorada como uma alternativa para análise simultânea de nitrito e nitrato com eletrodo compósito de grafite e zeólita dopado com prata. ${ }^{101}$ Dois pulsos de potenciais foram aplicados sequencialmente. Em $\mathrm{E}_{1}$, nitrato é reduzido e em $\mathrm{E}_{2}$, nitrito é oxidado. A técnica possibilitou a detecção direta e simultânea em amostras com nitrato na faixa de concentração entre 1,0 e $10 \mathrm{mmol} \mathrm{L}^{-1}$ e de nitrito entre 0,1 e $1,0 \mathrm{mmol} \mathrm{L}$. Os limites de detecção calculados foram 0,6 e $0,01 \mathrm{mmol} \mathrm{L}^{-1}$ para nitrato e nitrito, respectivamente. Os resultados obtidos nos estudos de recuperação em soluções aquosas foram próximos a $100 \%$, com desvio padrão relativo de 3,8 e 3,2\% para nitrato e nitrito, respectivamente. O método proposto no modo MPA é rápido, de baixo custo e não requer qualquer preparação da amostra. 


\section{Uso do método de padrão interno em sistema FIA com detecção por MPA}

Apesar do sistema FIA com detecção amperométrica pulsada apresentar vantagens frente aos métodos eletroquímicos estacionários ou em relação à amperometria convencional, a reprodutibilidade de resposta pode ser afetada por erros aleatórios como, por exemplo, variações na vazão, na temperatura, no volume de injeção, diferença na força iônica entre as soluções padrões e amostras e inserções acidentais de bolhas de ar na solução transportadora. Além disto, uma inconveniência da detecção amperométrica usando eletrodos sólidos é a perda de reprodutibilidade, devido à adsorção de produtos ou impurezas na superfície dos eletrodos sólidos, principalmente, quando o uso de pulsos de potenciais de limpeza apresenta limitações para manutenção e/ou renovação desta superfície. .,102 $^{2}$

A possibilidade de quantificação de dois analitos simultaneamente por FIA com detecção amperométrica usando um único eletrodo de trabalho permitiu a Gimenes et al. demonstrarem, de forma inédita, a implementação do método do padrão interno em sistemas FIA com detecção amperométrica. ${ }^{103} \mathrm{O}$ método do padrão interno foi introduzido aplicando uma estratégia similar à utilizada na determinação simultânea discutida anteriormente. ${ }^{98,99}$ Uma sequência de pulsos de potenciais foi aplicada ao eletrodo de trabalho para detectar simultaneamente o analito de interesse e o composto usado como padrão interno. Assim, as correntes do analito e do padrão interno são afetadas similarmente por variações em parâmetros como vazão, volume de injeção, temperatura e força iônica, bem como na inserção acidental de bolhas de ar e por alterações que ocorram na superfície do eletrodo de trabalho no transcorrer do experimento. A viabilidade do método foi demonstrada usando ácido úrico como analito e $\left[\mathrm{Fe}(\mathrm{CN})_{6}{ }^{3-}\right]$ como padrão interno. A seguinte sequência de pulsos de potenciais foi aplicada ao eletrodo de trabalho de carbono vítreo para esta finalidade: $+0,80 \mathrm{~V} / 300 \mathrm{~ms}$ : somente o ácido úrico é oxidado e determinado sem a interferência de $\left[\mathrm{Fe}(\mathrm{CN})_{6}^{3-}\right]$ (padrão interno); +0,60 V/500 ms: remoção da interface eletrodo-solução do produto de oxidação do ácido úrico gerado em + 0,80 V; -0,05 V/400 ms: somente o $\left[\mathrm{Fe}(\mathrm{CN})_{6}{ }^{3-}\right]$ (padrão interno) é detectado por redução sem a interferência do ácido úrico (analito); +0,60 V/500 ms: remoção da interface eletrodo-solução do produto de redução do $\left[\mathrm{Fe}(\mathrm{CN})_{6}{ }^{3-}\right]$ gerado em $-0,05 \mathrm{~V}$.

Conforme relatado nesse trabalho, o método do padrão interno pode gerar uma melhora considerável na precisão do método, pois a influência de variações nos parâmetros citados anteriormente pode ser corrigida com base nas variações (de mesma intensidade) observadas no sinal do padrão interno. Desta forma, este método pode neutralizar uma das principais limitações de detectores amperométricos sólidos, que é a variação da resposta em função da gradual passivação ou contaminação da superfície do eletrodo de trabalho no transcorrer do experimento.

\section{ASPECTOS RELEVANTES DA DETECÇÃO MPA EM FIA}

A breve revisão apresentada sobre FIA com detecção amperométrica pulsada não deixa dúvidas que a técnica apresenta grande potencialidade para o desenvolvimento de métodos de análise com as seguintes características: alta estabilidade, boa seletividade e sensibilidade, alta frequência de amostragem, baixo custo de instrumentação e simplicidade nas etapas de tratamento das amostras, diminuindo significativamente a geração de resíduos. No entanto, informações sobre os fundamentos teóricos da técnica raramente são abordadas na literatura, o que em parte explica a escassa exploração da técnica no desenvolvimento de métodos de análise. Diante deste contexto, alguns parâmetros otimizáveis da técnica, como a variação da vazão do sistema, do volume de amostragem e do tempo de duração dos pulsos de potenciais aplicados serão discutidos neste trabalho com a finalidade de contribuir na popularização da técnica.

\section{Variação da vazão}

A Figura 1 apresenta os resultados obtidos na detecção de ferricianeto de potássio (espécie com comportamento reversível) por amperometria convencional e amperometria pulsada em função da variação da vazão do sistema. Os demais parâmetros foram mantidos constantes.

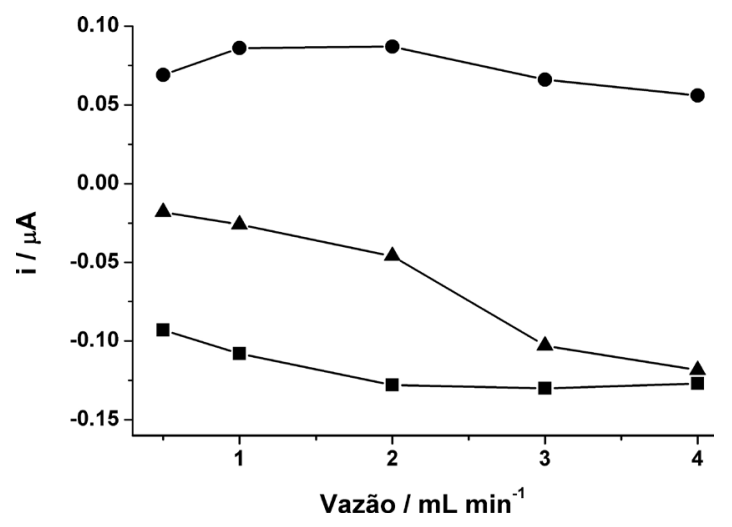

Figura 1. Magnitude da corrente medida no sistema FIA com detecção amperométrica em função da vazão da solução transportadora: ( $\mathbf{\Delta})$ amperometria convencional $(-0,05 \mathrm{~V})$; ( $\mathbf{\square})$ amperometria pulsada - pulso gerador (-0,05 $\mathrm{V} / 50 \mathrm{~ms})$; (-) amperometria pulsada-pulso coletor $(0,30 \mathrm{~V} / 50 \mathrm{~ms})$; analito: ferricianeto (75 $\left.\mu \mathrm{mol} \mathrm{L}^{-1}\right)$; eletrodo de trabalho: carbono vítreo $(3 \mathrm{~mm}$ de diâmetro). As demais variáveis foram mantidas constantes

Na detecção direta de ferricianeto (redução; -0,05 V), a magnitude da resposta eletroquímica aumenta com o incremento na vazão, tanto na amperometria convencional $(\boldsymbol{\Delta})$, como na amperometria pulsada (ם). O aumento no sinal é proporcionado pelo aumento da taxa de transporte de massa por unidade de tempo para a superfície do eletrodo, devido à diminuição da espessura da camada de difusão de Nernst (solução estagnada) em função do aumento do fluxo da solução. Desta forma, a distância que o material percorre nas imediações da superfície do eletrodo por difusão tende a diminuir em função do aumento da vazão do sistema. Porém, a partir de uma determinada vazão, a espessura da camada de difusão de Nernst deixa de diminuir e a corrente tende a se tornar constante, mesmo com novo incremento na vazão.

Apesar da corrente aumentar em função do aumento na vazão tanto na amperometria convencional como na pulsada, duas diferenças podem ser observadas: em vazões menores, a corrente medida na amperometria pulsada é muito superior à medida na amperometria convencional, tendendo a ser semelhante em vazões mais elevadas; a corrente medida na amperometria pulsada é menos dependente da variação da vazão. Para entender este fenômeno, consideremos o comportamento eletroquímico do ferricianeto apresentado na Equação 1.

$$
\left[\mathrm{Fe}(\mathrm{CN})_{6}\right]^{3-}+\mathrm{e}^{-} \leftrightarrow\left[\mathrm{Fe}(\mathrm{CN})_{6}\right]^{4-}
$$

$\mathrm{Na}$ amperometria convencional, somente a redução do $\left[\mathrm{Fe}(\mathrm{CN})_{6}\right]^{3-}$ é medida, enquanto que na amperometria pulsada, tanto o $\left[\mathrm{Fe}(\mathrm{CN})_{6}\right]^{3-}$ (pulso gerador; -0,05 V/50 ms), como o $\left[\mathrm{Fe}(\mathrm{CN})_{6}\right]^{4-}$ (pulso coletor; $+0,30 \mathrm{~V} / 50 \mathrm{~ms}$ ) são detectados (Figura 1). Em vazões baixas, a corrente medida na redução do $\left[\mathrm{Fe}(\mathrm{CN})_{6}\right]^{3-}$ é superior na amperometria pulsada, devido ao fenômeno da realimentação contínua. As 
espécies geradas no pulso de potencial anterior permanecem próximas à superfície do eletrodo por tempo suficiente para serem reduzidas ou oxidadas novamente e, por vezes, a mesma espécie pode passar por este processo mais de uma vez. À medida que o fluxo da solução vai aumentando, o fenômeno da realimentação vai diminuindo (remoção mais rápida do produto gerado) e a corrente de redução do $\left[\mathrm{Fe}(\mathrm{CN})_{6}\right]^{3-}$ (detecção direta) vai aumentando, o que explica a menor variação na corrente no pulso de potencial gerador (amperometria pulsada) em função da vazão.

Considerando somente o pulso de potencial coletor $(+0,30 \mathrm{~V} / 50$ $\mathrm{ms})$, no qual o produto gerado $\left(\left[\mathrm{Fe}(\mathrm{CN})_{6}\right]^{4-}\right)$ é detectado, podemos observar que inicialmente há um aumento na corrente medida em função do aumento na vazão e, a partir de determinada vazão, a corrente passa a diminuir. $\mathrm{O}$ aumento inicial na corrente ocorre em função de dois fatores: maior número de espécies geradas no pulso de potencial gerador e, o tempo de aplicação do pulso de potencial coletor é suficientemente rápido para detectar as espécies geradas no pulso anterior antes que elas difundam ou sejam removidas (pelo fluxo de solução) das imediações do eletrodo. Em vazões mais elevadas, o sinal analítico decresce, devido à remoção das espécies eletroquimicamente geradas nas proximidades do eletrodo (escala nanométrica) se tornar mais rápida do que a detecção no potencial coletor. É importante destacar que a vazão (elevada ou reduzida) é dependente das dimensões internas das tubulações do sistema FIA e da configuração do detector (neste caso, do tipo wall-jet). Além disto, a corrente de realimentação é dependente da área do eletrodo (neste caso, de $3 \mathrm{~mm}$ de diâmetro). Desta forma, experimentos utilizando células ou eletrodos de áreas ou geometrias diferentes deverão apresentar resultados que diferem dos apresentados aqui.

\section{Variação do volume de injeção}

A alça de amostragem é um compartimento que contém o volume de amostra ou solução padrão a ser inserido no percurso analítico através de um injetor. Em sistemas FIA, normalmente, as alças utilizadas têm capacidade entre 10 e $500 \mu \mathrm{L}$. Em estudos associando FIA e detecção amperométrica é bastante comum utilizar-se a altura do pico como medida analítica. Deste modo, quando se aumenta o volume da alça de amostragem, a magnitude do sinal vai aumentando até alcançar um limite máximo e constante. Como a detecção indireta (detecção em um potencial coletor) através da técnica MPA depende da quantidade gerada em um processo eletroquímico prévio, o comportamento da magnitude do sinal amperométrico em função da variação da alça de amostragem é semelhante à detecção direta de um composto eletroativo. Quando sistemas de análise em fluxo convencionais são utilizados, quanto menor o volume da alíquota injetada, menor a magnitude do sinal amperométrico, uma vez que a dispersão da amostra ocorre ao longo do percurso entre o injetor e a célula eletroquímica e no interior da mesma. A dispersão é uma característica intrínseca das análises em sistemas por injeção em fluxo ${ }^{104}$ que ocorrem em função de um gradiente de concentração da espécie química de interesse nas duas extremidades da zona da amostra, que se amplia à medida que aumenta o percurso entre o injetor e o detector. A dispersão do analito nas extremidades da zona da amostra causará maior efeito de diluição quanto menor for o volume da alíquota injetada (mantendo-se o percurso analítico fixo). No entanto, há um limite máximo de corrente dado pela altura de pico para um composto eletroativo em relação ao aumento do volume na alça de amostragem. A partir de certo volume, a diluição que ocorre nas extremidades da zona da amostra deixa de alcançar a região central da mesma e um sinal analítico constante é adquirido em relação à altura de pico a partir deste volume. Em contrapartida, se for avaliada a área em sistemas por injeção em fluxo, quanto maior a alça de amostragem, maior será a área do pico do composto investigado, não se observando um valor limite de corrente nesta condição. Deve-se ressaltar que, mesmo havendo um ganho na resposta amperométrica com o aumento do volume da alça de amostragem, a injeção de um volume maior de amostra implica em frequências de amostragem menores (maior tempo necessário para que a zona de amostra passe pelo detector) e maior consumo de amostra e reagentes. Além disso, na detecção eletroquímica, uma quantidade elevada de amostra pode agravar problemas de passivação ou contaminação da superfície do eletrodo, o que pode vir a prejudicar a reprodutibilidade dos resultados.

Outra questão que sempre deve ser observada na utilização da MPA em FIA está relacionada com a aquisição do número mínimo de pontos da corrente para cada pico transiente gerado numa injeção de amostra ou solução padrão. A aquisição deve ser rápida o suficiente para que pelo menos um ponto seja adquirido na altura máxima do pico. A escolha deste parâmetro é dependente da vazão, volume da alça de amostragem e velocidade de aquisição. Em estudos de otimização (não divulgados na literatura) foi verificado que um pico é adquirido sem deformação significativa quando este apresentar no mínimo 20 pontos. Em outras palavras, o sinal de corrente deve ser adquirido pelo menos 20 vezes durante a passagem da alíquota da amostra ou solução padrão pelo detector. Como exemplo, consideremos hipoteticamente que os seguintes pulsos de potenciais fossem aplicados em uma determinada análise: $+0,40 \mathrm{~V} / 0,10 \mathrm{~s} ;+0$, $80 \mathrm{~V} / 0,60 \mathrm{~s} ; 0,00 \mathrm{~V} / 0,03 \mathrm{~s} \mathrm{e}-0,10 \mathrm{~V} / 0,60 \mathrm{~s}$. A soma total do tempo dos pulsos de potenciais é de $1,33 \mathrm{~s}$, o que significa que um ponto é adquirido em cada amperograma (4 no total) a cada 1,33 s (software GPES - Eco Chemie). Portanto, para que um pico transiente apresente pelo menos 20 pontos de aquisição de corrente, a zona de amostra deve levar no mínimo $27 \mathrm{~s}$ para passar pelo detector. Este tempo de contato é controlado pela dimensão da alça de amostragem e pela vazão. Apesar de haver a necessidade deste cuidado, nas condições hipotéticas acima é possível realizar mais de 120 injeções/h, o que ainda pode ser considerado uma alta frequência de amostragem comparada a outros métodos de análise.

\section{Variação do tempo de duração do pulso de potencial}

A corrente amperométrica monitorada durante a aplicação de cada pulso de potencial é governada por dois componentes de corrente: a corrente faradaica, $\mathrm{I}_{\mathrm{F}}$ (devido ao processo de transferência de carga no eletrodo) e a corrente capacitiva, $\mathrm{I}_{\mathrm{C}}$ (originária do carregamento da dupla camada elétrica quando um pulso de potencial é aplicado). ${ }^{105} \mathrm{~A}$ participação da $\mathrm{I}_{\mathrm{F}}$ na corrente amperométrica total depende da presença de espécies eletroativas que podem ser oxidadas ou reduzidas na superfície do eletrodo, sob o pulso de potencial aplicado.

Ao aplicar um pulso de potencial na presença de material eletroativo, os processos faradaico $\left(\mathrm{I}_{\mathrm{F}}\right)$ e capacitivo $\left(\mathrm{I}_{\mathrm{C}}\right)$ ocorrerão simultaneamente. A componente $\mathrm{I}_{\mathrm{C}}$ é alta no início da aplicação do pulso de potencial, mas diminui rapidamente (de forma exponencial) com o tempo da aplicação do pulso $\left(\mathrm{I}_{\mathrm{C}} \alpha \mathrm{e}^{-\mathrm{t}}\right)$. Para uma solução que contém um analito eletroativo, a dependência da componente $\mathrm{I}_{\mathrm{F}}$ com o tempo de aplicação do pulso depende da velocidade do transporte de massa do analito ao eletrodo. Sob condições estacionárias, $\mathrm{I}_{\mathrm{F}}$ diminui mais lentamente que a $\mathrm{I}_{\mathrm{C}}$ em função do tempo de aplicação do pulso de potencial $\left(\mathrm{I}_{\mathrm{F}} \alpha \mathrm{t}^{-1 / 2}\right){ }^{105}$ Desta forma, quanto maior o tempo de aplicação do pulso de potencial, menor será a contribuição da $I_{C}$ para a corrente total monitorada, uma vez que a aquisição de corrente é realizada próximo do final do pulso de potencial.

Entretanto, deve-se ressaltar que a $\mathrm{I}_{\mathrm{C}}$ é inversamente proporcional ao tempo de aplicação de cada pulso e diretamente proporcional à variação do degrau de potencial. Com isto, pulsos de potenciais 
aplicados por curtos períodos de tempo ou de grande incremento geram $\mathrm{I}_{\mathrm{C}}$ elevadas. Esta contribuição, em princípio, não afeta o sinal amperométrico de interesse $\left(\mathrm{I}_{\mathrm{F}}\right)$, uma vez que a corrente em sistemas FIA é medida continuamente, antes, durante e após a passagem da zona da amostra. Dessa forma, a contribuição da $\mathrm{I}_{\mathrm{C}}$ normalmente é a mesma, antes, durante e após a passagem da zona da amostra que contém o analito de interesse. Consequentemente, durante a passagem da zona da amostra é possível monitorar apenas a contribuição da $\mathrm{I}_{\mathrm{F}}$ para o sinal amperométrico. Entretanto, o aumento excessivo da $\mathrm{I}_{\mathrm{C}}$ (uso de pulsos de potenciais de amplitude elevada) proporciona um acréscimo no sinal de corrente de fundo (e aumento proporcional no ruído da linha base) resultando no aumento do limite de detecção para a espécie eletroativa investigada. A Figura 2 mostra a dependência do sinal de $\mathrm{I}_{\mathrm{F}}$ e $\mathrm{I}_{\mathrm{C}}$ em função do tempo de aplicação de um pulso de potencial em um sistema estacionário (Figura 2a) e em sistemas FIA sob vazões baixas (Figura 2b) e mais elevadas (Figura 2c).

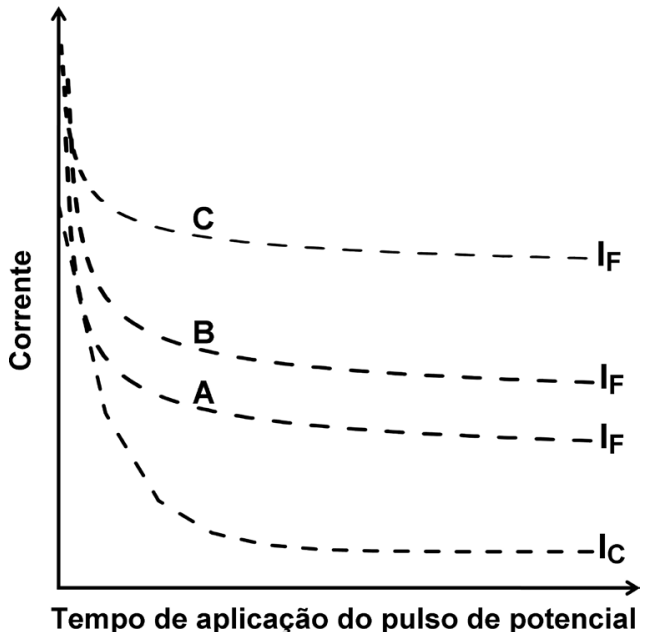

Figura 2. Dependência da corrente faradaica $\left(I_{F}\right)$ e da corrente capacitiva $\left(I_{C}\right)$ em função do tempo de aplicação de um pulso de potencial em uma solução em estado estacionário (A) e em sistemas em fluxo com vazões baixas $(B)$ e mais elevadas $(C)$

A Figura 2 demonstra que $\mathrm{I}_{\mathrm{C}}$ tende a diminuir à medida que os tempos de aplicação dos pulsos de potenciais aumentam, sendo que a magnitude da redução da $\mathrm{I}_{\mathrm{C}}$ é a mesma, tanto no sistema estacionário (Figura 2a) quanto em sistemas em fluxo com vazões baixas (Figura 2b) e mais elevadas (Figura 2c). Entretanto, a $\mathrm{I}_{\mathrm{F}}$ apresenta uma diminuição mais acentuada em sistemas estacionários (Figura 2a) do que em sistemas hidrodinâmicos (Figuras $2 \mathrm{~b}$ e c), tendendo a diminuir menos quanto maior for à vazão utilizada. Este fenômeno ocorre com a $\mathrm{I}_{\mathrm{F}}$ devido ao aumento da taxa de reposição das espécies eletroativas na superfície do eletrodo em função do aumento do transporte de material, conforme já discutido anteriormente. O comportamento apresentado na Figura 2 é comum ao pulso de potencial gerador (detecção direta) e ao coletor (detecção indireta) em se tratando de $\mathrm{I}_{\mathrm{C}}$. Em relação à $\mathrm{I}_{\mathrm{F}}$, a Figura 2 corresponde ao fenômeno que ocorre somente no pulso de potencial gerador. Em vazões suficientemente elevadas, um sinal de $\mathrm{I}_{\mathrm{F}}$ constante poderá ser observado independentemente do tempo de aplicação do pulso de potencial gerador, pois a reposição do material consumido na interface eletrodo/solução será rápida o suficiente para evitar a polarização por concentração.

Em relação ao pulso de potencial coletor (detecção de um produto gerado em um pulso de potencial anterior) a dependência da corrente faradaica em função do tempo de aplicação do pulso de potencial é diferente, conforme pode ser observado na Figura 3. O sinal de aquisição máximo, neste caso, é obtido quando os tempos de aquisição são curtos, pois o produto gerado tende a ser removido da superfície do eletrodo pelo fluxo da solução. ${ }^{85,98}$ Por outro lado, quando a intenção não é detectar um determinado produto gerado, pode-se optar por aumentar o tempo de aplicação do pulso de potencial coletor ${ }^{99}$ ou ainda inserir pulsos de potenciais intermediários que contribuem no consumo do produto gerado. ${ }^{103}$

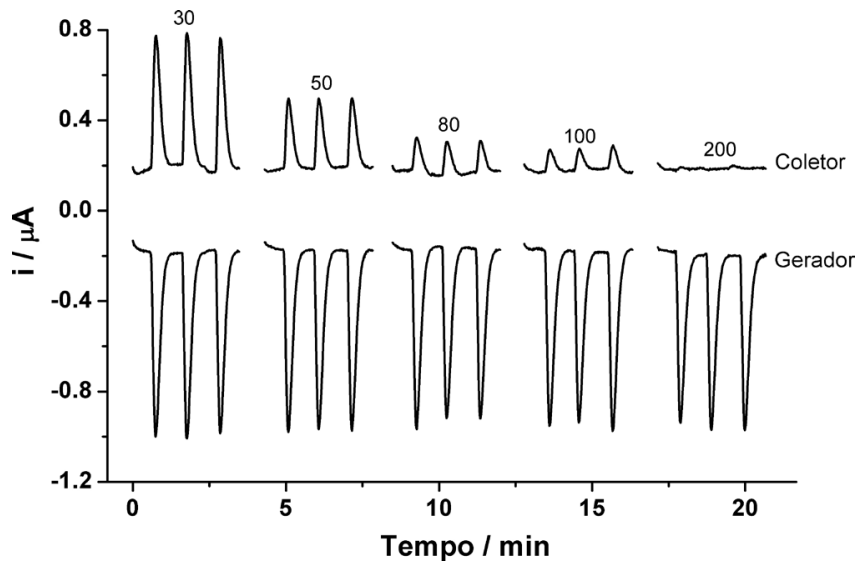

Figura 3. Dependência da corrente faradaica em função do tempo de aplicação do pulso de potencial coletor $(0,30 \mathrm{~V} ; 30$ a $200 \mathrm{~ms})$. Pulso de potencial gerador: -0,05 V/ $400 \mathrm{~ms}$; vazão: 2,0 $\mathrm{mL} \mathrm{min}^{-1}$; volume injetado: $300 \mu \mathrm{L}$; eletrodo de trabalho: carbono vítreo $(\varnothing=3 \mathrm{~mm})$; eletrólito: tampão ácido acético/acetato 0,1 $\mathrm{mol} \mathrm{L}^{-1}$; analito: ferricianeto (75 $\mu \mathrm{mol} \mathrm{L^{-1 } )}$

Outro ponto a ser considerado (para melhorar a sensibilidade), quando a intenção é monitorar um produto gerado (detecção indireta), é o tempo de aplicação do pulso de potencial gerador. A Figura 4 apresenta a dependência da magnitude do sinal amperométrico no pulso de potencial coletor (mantido num tempo de aplicação mínino e constante, $30 \mathrm{~ms}$ ) em função do tempo de aplicação do pulso de potencial gerador.

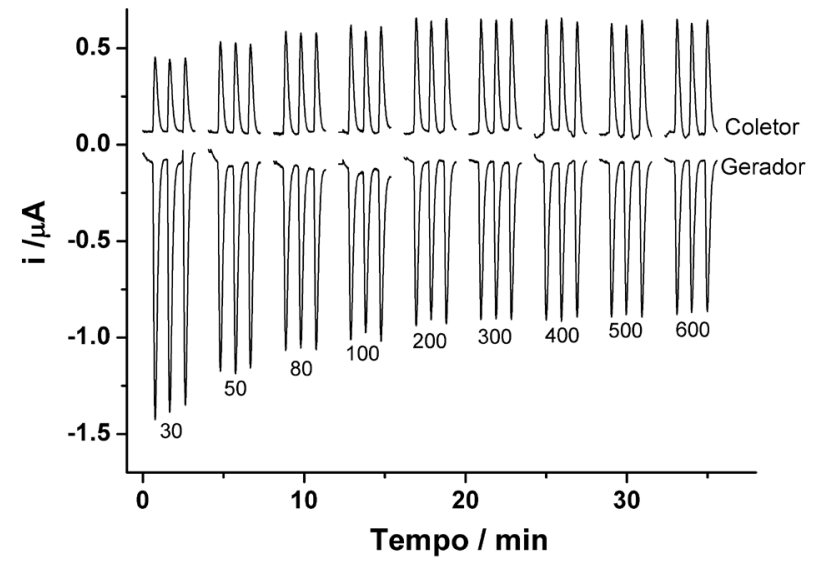

Figura 4. Dependência da corrente faradaica em função do tempo de aplicação do pulso de potencial gerador (-0,05 V; 30 a $600 \mathrm{~ms})$. Pulso de potencial coletor: $+0,30 \mathrm{~V} / 30 \mathrm{~ms}$; demais parâmetros conforme Figura 3

Na Figura 4 fica evidente que a intensidade do sinal no pulso de potencial coletor sofre influência do tempo de aplicação do pulso de potencial gerador. Quando este pulso é aplicado durante períodos de tempo muito curtos (cerca de $30 \mathrm{~ms}$ ), o sinal de corrente no pulso de potencial coletor é menor. Com o aumento no tempo de aplicação do pulso de potencial gerador, o sinal amperométrico no pulso de potencial coletor aumenta até um dado tempo, a partir do qual passa a ser constante (200 ms, Figura 4, sob vazão de 2,0 $\left.\mathrm{mL} \mathrm{min}{ }^{-1}\right)$. O 
aumento inicial do sinal detectado no pulso de potencial coletor está relacionado com a quantidade crescente de produto formado na interface eletrodo-solução no pulso de potencial gerador, em função do aumento no tempo de aplicação do pulso. É difícil prever a magnitude da corrente medida nestes pulsos de potenciais, pois são dependentes da vazão, da viscosidade do meio, da área do eletrodo, da geometria da célula e dos coeficientes de difusão das espécies envolvidas, entre outros. Outro fator importante que deve ser considerado é quando a espécie gerada adsorve na superfície do eletrodo. Neste caso, o aumento da corrente de pico no pulso de potencial coletor em função do aumento do tempo do pulso de potencial gerador é mais acentuado do que para espécies que não adsorvem no eletrodo de trabalho.

\section{CONCLUSÕES E PERSPECTIVAS}

Este trabalho apresentou as aplicações e potencialidades da detecção eletroquímica de amperometria de múltiplos pulsos (MPA). A utilização desta técnica associada a sistemas FIA pode proporcionar aplicações analíticas simples, rápidas, com baixo custo de instrumentação e que necessitam de etapas mínimas de preparação da amostra (dissolução e diluição). Os trabalhos descritos na literatura que utilizaram a técnica MPA acoplada à FIA apresentaram boa sensibilidade, seletividade e reprodutibilidade. Cabe destacar que, na maioria dos estudos em que a técnica MPA foi explorada, ficou demonstrado que os eletrodos de trabalho podem ser utilizados sem qualquer modificação química ou eletroquímica de sua superfície, o que torna o método mais atraente para aplicações em análises de rotina. Adicionalmente, quando a limpeza eletroquímica não for eficiente (limitação da técnica), a implementação do método do padrão interno ${ }^{103}$ pode contribuir para manter a precisão dos resultados obtidos, mesmo com a progressiva contaminação ou passivação do eletrodo usado.

A técnica MPA também apresenta limitações. No uso de pulsos de potenciais de grande amplitude, a corrente capacitiva pode ser muito maior que a faradaica e os resultados podem se tornar irreprodutíveis na determinação de espécies químicas em nível de traços. Neste caso, o uso de eletrodos de diamante dopado com boro parece promissor, devido sua elevada relação corrente faradaica/corrente capacitiva. Outro problema que pode ocorrer quando uma sequência cíclica de pulsos de potenciais é aplicada em função do tempo está relacionado ao produto gerado em um pulso de potencial anterior, o qual pode ser um interferente em um pulso de potencial subsequente.

Apesar destas limitações, a detecção MPA acoplada a sistemas FIA tem um grande potencial a ser explorado, principalmente em relação à estabilidade do sinal amperométrico em função do tempo (limpeza eletroquímica ou uso do padrão interno) e na possibilidade do desenvolvimento de novos métodos que permitam a análise simultânea de espécies presentes em diferentes amostras. Para concluir, as discussões apresentadas neste trabalho visam contribuir para a popularização da técnica de amperometria de múltiplos pulsos (MPA).

\section{AGRADECIMENTOS}

Ao apoio financeiro do CNPq (processo no 476269/2010-5), da CAPES, FAPEMIG e FAPESP.

\section{REFERÊNCIAS}

1. Ruzicka, J.; Hansen, E. H.; TrAC, Trends Anal. Chem. 2008, 27, 390.

2. Mervartova, K.; Polasek, M.; Calatayud, J. M.; J. Pharm. Biomed. Anal. 2007, 45, 367.

3. Chailapakul, O.; Ngamukot, P.; Yoosamran, A.; Siangproh, W.; Wangfuengkanagul, N.; Sensors 2006, 6, 1383.
4. Miro, M.; Frenzel, W.; Microchim. Acta 2004, 148, 1.

5. Dunec, A. F.; Cheregi, M.; Calatayud, J. M.; Mateo, J. V. G.; Enein, H. Y. A.; Crit. Rev. Anal. Chem. 2003, 33, 57.

6. Hansen, E. H.; Wang, J. H.; Anal. Lett. 2004, 37, 345.

7. Danet, A. F.; Cheregi, M.; Calatayud, J. M.; Mateo, J. V. G.; Enein, H. Y. A.; Crit. Rev. Anal. Chem. 2001, 31, 191.

8. Ruzicka, J.; Hansen, E. H.; Anal. Chim. Acta 1975, 78, 145.

9. Ruzicka, J.; Hansen, E. H.; Anal. Chim. Acta 1978, 99, 37.

10. Alonso, E. V.; de Torres, A. G.; Pavon, J. M. C.; Talanta 2001, 55, 219.

11. Begak, O. Y.; Borodin, A. V.; Ind. Lab. 1998, 64, 371.

12. Perez-Ruiz, T.; Lozano, C. M.; Tomas, V.; Ruiz, E.; Microchim. Acta 2007, 158, 299.

13. Takayanagi, T.; Yamashita, H.; Motomizu, S.; Musijowski, J.; Trojanowicz, M.; Talanta 2008, 74, 1224.

14. Baezzat, M. R.; Izadpanah, M.; Luminescence 2008, 23, 59.

15. Yu, J.; Zhang, C.; Tan, Y.; Ge, S.; Dai, P.; Zha, Y.; Luminescence 2008, 23, 107

16. Couto, C.; Montenegro, M.; Quim. Nova 2000, 23, 774.

17. Hu, A.; Dessy, R. E.; Graneli, A.; Anal. Chem. 1983, 55, 320.

18. Ivaska, A.; Ryan, T. H.; Collect. Czech. Chem. Commun. 1981, 46, 2865.

19. Agui, L.; Guzman, A.; Pedrero, M.; Yanez-Sedeno, P.; Pingarron, J. M.; Electroanalysis 2003, 15, 601.

20. Shitanda, I.; Takamatsu, S.; Watanabe, K.; Itagaki, M.; Electrochim. Acta 2009, 54, 4933.

21. Wei, Y. Y.; Li, Y.; Qu, Y. H.; Xiao, F.; Shi, G. Y.; Jin, L. T.; Anal. Chim. Acta 2009, 643, 13

22. Johnson, D. C.; Weber, S. G.; Bond, A. M.; Wightman, R. M.; Shoup, R. E.; Krull, I. S.; Anal. Chim. Acta 1986, 180, 187.

23. Trojanowicz, M.; Szewczynska, M.; Wcislo, M.; Electroanalysis $\mathbf{2 0 0 3}$, 15,347 .

24. Yashin, Y. I.; Yashin, A. Y.; J. Anal. Chem. 2004, 59, 1121.

25. Galli, A.; De Souza, D.; Garbellini, G. S.; Coutinho, C. F. B.; Mazo, L. H.; Avaca, L. A.; Machado, S. A. S.; Quim. Nova 2006, 29, 105.

26. Catarino, R. I. L.; Conceicão, A. C. L.; Garcia, M. B. Q.; Goncalves, M. L. S.; Lima, J.; dos Santos, M. M. C.; J. Pharm. Biomed. Anal. 2003, 33, 571.

27. de Carvalho, R. M.; Freire, R. S.; Rath, S.; Kubota, L. T.; J. Pharm. Biomed. Anal. 2004, 34, 871.

28. Skoog, D. A.; Holler, F. J.; Nieman, T. A.; Princípios de Análise Instrumental, $5^{\mathrm{a}}$ ed., Bookman Companhia Editora: Porto Alegre, 2002.

29. Paixao, T.; Matos, R. C.; Bertotti, M.; Electroanalysis 2003, 15, 1884.

30. Matos, R. C.; Angnes, L.; Araujo, M. C. U.; Saldanha, T. C. B.; Analyst 2000, 125, 2011.

31. Palaharn, S.; Charoenraks, T.; Wangfuengkanagul, N.; Grudpan, K.; Chailapakul, O.; Anal. Chim. Acta 2003, 499, 191.

32. Giuriati, C.; Cavalli, S.; Gorni, A.; Badocco, D.; Pastore, P.; J. Chromatogr., A 2004, 1023, 105.

33. Guzman, A.; Agui, L.; Pedrero, M.; Yanez-Sedeno, P.; Pingarron, J. M.; J. Pharm. Biomed. Anal. 2003, 33, 923.

34. Cai, Y. E.; Cai, Y. Q.; Shi, Y. L.; Mou, S.; Lu, Y. Q.; J. Chromatogr., A 2006, 1118,35

35. Johnson, D. C.; Abst. Pap. Am. Chem. Soc. 1981, 182, 42.

36. Agui, M. L.; Calavia, E.; Yanezsedeno, P.; Pingarron, J. M.; Anal. Chim. Acta 1995, 305, 324.

37. Dilleen, J. W.; Lawrence, C. M.; Slater, J. M.; Analyst 1996, 121, 755.

38. Lee, J. W.; Yeo, I. H.; Pyun, C. H.; Bul. Korean Chem. Soc. 1997, 18, 316.

39. Polta, J. A.; Johnson, D. C.; Anal. Chem. 1985, 57, 1373.

40. Neuburger, G. G.; Johnson, D. C.; Anal. Chem. 1987, 59, 203.

41. Larew, L. A.; Mead, D. A.; Johnson, D. C.; Anal. Chim. Acta 1988, 204, 43.

42. de Silva, J. A. F.; Quim. Nova 2003, 26, 56.

43. Trojanowicz, M.; Anal. Chim. Acta 2009, 653, 36. 
44. Garcia, C. D.; Henry, C. S.; Anal. Chem. 2003, 75, 4778.

45. Jensen, M. B.; Abst. Pap. Am. Chem. Soc. 2001, 222, 379.

46. Weber, G.; Neumann, G.; Romheld, V.; Anal. Bioanal. Chem. 2002, 373, 767.

47. Cao, X. N.; Li, J. H.; Xu, H. H.; Lin, L.; Xian, Y. Z.; Yamamoto, K.; Jin, L. T.; Biomed. Chromatogr. 2004, 18, 564.

48. Charoenraks, T.; Chuanuwatanakul, S.; Honda, K.; Yamaguchi, Y.; Chailapakul, O.; Anal. Sci. 2005, 21, 241.

49. Gonzaga, F. B.; Pereira, C. F.; Guarita-Santos, A. J. M.; De, J. R. S.; Electroanalysis 2005, 17, 2084.

50. Hanko, V. P.; Rohrer, J. S.; Liu, H. H.; Zheng, C.; Zhang, S.; Liu, X.; Tang, X.; J. Pharm. Biomed. Anal. 2008, 47, 828.

51. Jeong, J. S.; Kwon, H.; Yoon, H. R.; Lee, Y. M.; Choi, T. Y.; Hong, S. P.; Anal. Biochem. 2008, 376, 200.

52. Joo, K. M.; Park, C. W.; Jeong, H. J.; Lee, S. J.; Chang, I. S.; J. Chromatogr, B: Anal. Technol. Biomed. Life Sci. 2008, 865, 159.

53. Kwon, H. J.; Jeong, J. S.; Lee, Y. M.; Hong, S. P.; J. Chromatogr., A 2008, 1185, 251

54. Raessler, M.; Wissuwa, B.; Breul, A.; Unger, W.; Grimm, T.; J. Agric. Food Chem. 2008, 56, 7649.

55. Toropainen, T.; Jarho, P.; Lehtonen, M.; Keski-Rahkonen, P.; Raatikainen, H.; Jarvinen, T.; J. Chromatogr., B: Anal. Technol. Biomed. Life Sci. 2008, 867, 90.

56. Jeon, M. H.; Kwon, H. J.; Jeong, J. S.; Lee, Y. M.; Hong, S. P.; J. Chromatogr., A 2009, 1216, 4568.

57. Kwon, H. J.; Jeong, J. S.; Sim, H. J.; Lee, Y. M.; Kim, Y. S.; Hong, S. P.; J. Chromatogr., A 2009, 1216, 4445.

58. Dieker, J. W.; Vanderlinden, W. E.; Poppe, H.; Talanta 1979, $26,511$.

59. Hackman, M. R.; Brooks, M. A.; J. Chromatogr. 1981, 222, 179.

60. Hughes, S.; Johnson, D. C.; Anal. Chim. Acta 1981, 132, 11.

61. Mayer, W. J.; Greenberg, M. S.; J. Chromatogr. Sci. 1979, 17, 614.

62. Austinharrison, D. S.; Johnson, D. C.; Electroanalysis 1989, 1, 189.

63. Johnson, D. C.; Dobberpuhl, D.; Roberts, R.; Vandeberg, P.; J. Chromatogr. 1993, 640, 79.

64. Weber, P. L.; Lunte, S. M.; Electrophoresis 1996, 17, 302.

65. Rohrer, J. S.; Oligosaccharides Food Agric. 2003, 849, 16.

66. Jandik, P.; Cheng, J.; Avdalovic, N.; J. Biochem. Biophys. Methods 2004, $60,191$.

67. Bebeselea, A.; Manea, F.; Burtica, G.; Nagy, L.; Nagy, G.; Talanta 2010, $80,1068$.

68. Matysik, F. M.; Microchim. Acta 2008, 160, 1.

69. Montero, C. M.; Dodero, M. C. R.; Sanchez, D. A. G.; Barroso, C. G.; Chromatographia 2004, 59, 15.

70. Ozkan, S. A.; Uslu, B.; Aboul-Enein, H. Y.; Crit. Rev. Anal. Chem. 2003, $33,155$.

71. Garcia, C. D.; Henry, C. S.; Electroanalysis 2005, 17, 223.

72. Holland, L. A.; Lunte, S. M.; Anal. Commun. 1998, 35, 1 H.

73. Manica, D. P.; Mitsumori, Y.; Ewing, A. G.; Anal. Chem. 2003, 75, 4572.

74. Garcia, C. D.; Henry, C. S.; Electroanalysis 2005, 17, 1125.

75. Xu, J. J.; Wang, A. J.; Chen, H. Y.; TrAC, Trends Anal. Chem. 2007, 26, 125.

76. Felix, F. S.; Angnes, L.; J. Pharm. Sci. 2010, 99, 4784.
77. Ozkan, S. A.; Chromatographia 2007, 66, S3.

78. Johnson, D. C.; Lacourse, W. R.; Anal. Chem. 1990, 62, A589.

79. Cheng, J.; Jandik, P.; Liu, X.; Pohl, C.; J. Electroanal. Chem. 2007, 608, 117.

80. Gerlache, M.; Girousi, S.; Quarin, G.; Kauffmann, J. M.; Electrochim. Acta 1998, 43, 3467.

81. Vandaveer, W. R.; Pasas-Farmer, S. A.; Fischer, D. J.; Frankenfeld, C. N.; Lunte, S. M.; Electrophoresis 2004, 25, 3528.

82. Perez-Olmos, R.; Soto, J. C.; Zarate, N.; Araujo, A. N.; Montenegro, M.; Anal. Chim. Acta 2005, 554, 1.

83. Trojanowicz, M.; Anal. Chim. Acta 2011, 688, 8.

84. Possari, R.; Carvalhal, R. F.; Mendes, R. K.; Kubota, L. T.; Anal. Chim. Acta 2006, 575, 172.

85. Gimenes, D. T.; dos Santos, W. T. P.; Tormin, T. F.; Munoz, R. A. A.; Richter, E. M.; Electroanalysis 2010, 22, 74.

86. dos Santos, W. T. P.; Azevedo, E. F.; Richter, E. M.; de Albuquerque, Y. D. T.; Quim. Nova 2009, 32, 2412.

87. Tormin, T. F.; Gimenes, D. T.; Silva, L. G.; Ruggiero, R.; Richter, E. M.; Ferreira, V. S.; Munoz, R. A. A.; Talanta 2010, 82, 1599.

88. Hlabangana, L.; Hernandez-Cassou, S.; Saurina, J.; Curr. Pharm. Anal. 2006, $2,127$.

89. Tomsu, D.; Icardo, M. C.; Calatayud, J. M.; J. Pharm. Biomed. Anal. 2004, 36, 549 .

90. Shpigun, L. K.; Shushenachev, Y. V.; Kamilova, P. M.; Anal. Chim. Acta 2006, 573, 360 .

91. Li, Y. Q.; Hu, J. M.; Yang, J. G.; Zheng, B.; Ha, Y. Q.; Anal. Chim. Acta 2002, 461, 181.

92. Azubel, M.; Fernandez, F. M.; Tudino, M. B.; Troccoli, O. E.; Anal. Chim. Acta 1999, 398, 93.

93. Zhou, Y.; Li, Y. Q.; Yang, J. G.; Zheng, B.; Spectrosc. Spectral Anal. 2003, 23, 374

94. Yue, X. F.; Zhang, Z. Q.; J. Anal. Chem. 2007, 62, 992.

95. Li, Y. S.; Muo, Y.; Xie, H. M.; Anal. Chem. Acta 2002, 455, 315.

96. Suvardhan, K.; Kumar, K. S.; Babu, S. H.; Jayaraj, B.; Chiranjeevi, P.; Talanta 2008, 75, 1148.

97. Surareungchai, W.; Deepunya, W.; Tasakorn, P.; Anal. Chim. Acta 2001, $448,215$.

98. dos Santos, W. T. P.; de Almeida, E. G. N.; Ferreira, H. E. A.; Gimenes, D. T.; Richter, E. M.; Electroanalysis 2008, 20, 1878.

99. Santos, W. T. P.; Gimenes, D. T.; Almeida, E. G. N.; Eiras, S. D. P.; Albuquerque, Y. D. T.; Richter, E. M.; J. Braz. Chem. Soc 2009, 20, 1249.

100. Medeiros, R. A.; Lourencao, B. C.; Rocha, R. C.; Fatibello, O.; Anal. Chem. 2010, 82,8658 .

101. Manea, F.; Remes, A.; Radovan, C.; Pode, R.; Picken, S.; Schoonman, J.; Talanta 2010, 83, 66.

102. Yu, J. J.; Huang, W. G.; Hibbert, D. B.; Electroanalysis 1997, 9, 544.

103. Gimenes, D. T.; dos Santos, W. T. P.; Munoz, R. A. A.; Richter, E. M.; Electrochem. Commun. 2010, 12, 216.

104. Fox, R. W.; McDownald, A. T.; Introdução à Mecânica de Fluídos, Ed. Guanabara Dois: Rio de Janeiro, 1981

105. Bard, A. J.; Faulkner, L. R.; Electrochemical Methods, $1^{\text {a }}$ ed.; John Wiley \& Sons: New York, 1980, p. 391. 